\title{
Hybrid modeling of relativistic underdense plasma photocathode injectors
}

\author{
Y. Xi, ${ }^{1}$ B. Hidding, ${ }^{1,2}$ D. Bruhwiler, ${ }^{3}$ G. Pretzler, ${ }^{4}$ and J. B. Rosenzweig ${ }^{1}$ \\ ${ }^{1}$ Department of Physics and Astronomy, University of California, Los Angeles, California, USA \\ ${ }^{2}$ Institut für Experimentalphysik, Universität Hamburg \& DESY, 22607 Hamburg, Germany \\ ${ }^{3}$ University of Colorado at Boulder, 390UCB, Boulder, Colorado 80309, USA \\ ${ }^{4}$ Institut für Laser- und Plasmaphyik, Heinrich-Heine-Universität Düsseldorf, Germany
}

(Received 6 November 2012; published 25 March 2013)

\begin{abstract}
The dynamics of laser ionization-based electron injection in the recently introduced plasma photocathode concept is analyzed analytically and with particle-in-cell simulations. The influence of the initial few-cycle laser pulse that liberates electrons through background gas ionization in a plasma wakefield accelerator on the final electron phase space is described through the use of Ammosov-Deloine-Krainov theory as well as nonadiabatic Yudin-Ivanov (YI) ionization theory and subsequent downstream dynamics in the combined laser and plasma wave fields. The photoelectrons are tracked by solving their relativistic equations of motion. They experience the analytically described transient laser field and the simulationderived plasma wakefields. It is shown that the minimum normalized emittance of fs-scale electron bunches released in mulit-GV/m-scale plasma wakefields is of the order of $10^{-2} \mathrm{~mm} \mathrm{mrad}$. Such unprecedented values, combined with the dramatically increased controllability of electron bunch production, pave the way for highly compact yet ultrahigh quality plasma-based electron accelerators and light source applications.
\end{abstract}

DOI: 10.1103/PhysRevSTAB.16.031303

\section{INTRODUCTION}

Acceleration of charged particles in plasma is an extremely promising, emerging new method, as it can exploit accelerating and focusing electric fields that can straightforwardly reach tens of $\mathrm{GV} / \mathrm{m}$ or more [1], permitting extremely compact accelerators along with high current, short pulse, compact phase space or high brightness beams. These large fields arise due to the collective motion of electrons in relativistic phase-velocity plasma waves, which can be driven either by ultrashort intense, focused laser [2-4] or particle beams [5,6]. Such driving beams ideally have FWHM pulse lengths that are shorter than $\lambda_{\mathrm{p}} / 2$, where $\lambda_{\mathrm{p}}=2 \pi / k_{p}=\sqrt{\pi / n_{0} r_{e}}$ is the plasma wavelength, and $k_{p}$ is the plasma wave number, $n_{0}$ is the electron density, and $r_{e}$ is the classical radius of the electron. With sufficiently intense driving beams, the plasma electron displacement induced may result in completely electron-rarefied plasma blowout cavities that trail the driver pulse through the plasma, and support the desired large-amplitude plasma wakefields, having peak electric fields $E$ near "wave breaking," $E \sim E_{\mathrm{WB}}=k_{p} m_{e} c^{2} / e$, where $m_{e} c^{2}$ is the electron rest energy and $e$ is the electron charge [7]. This scenario stands in contrast to conventional metallic cavities, which are stationary in the laboratory frame, and thus a long array of such cavities are needed to accelerate to high energies.

Published by the American Physical Society under the terms of the Creative Commons Attribution 3.0 License. Further distribution of this work must maintain attribution to the author(s) and the published article's title, journal citation, and DOI.
PACS numbers: $52.38 . \mathrm{Kd}, 41.75 . \mathrm{Jv}, 32.80 . \mathrm{Fb}, 52.35 . \mathrm{Mw}$

On the other hand, plasma blowout cavities are stationary in the wave frame, which travels at nearly the speed of light $c$, meaning that only one propagating cavity is needed for long-range acceleration. It is possible to inject electrons directly into these moving cavities from the background plasma, in a scheme that bears both similarities and dramatic differences to analogous injection processes in stationary metallic cavities, which are termed photoinjectors, or rf photocathode guns.

Injection of background plasma electrons into the plasma wave, trapping them in the wave, and forming an electron bunch with compact six-dimensional phase space volume is a complex process presenting difficult challenges. Schemes that yield control over such characteristics, such as selfinjection [8-14], density step injection [15-19], multiple laser pulse injection [20-23], ionization-induced injection [24-28], and combinations of these have been proposed and developed to varying degrees in recent years. For applications such as driving an X-ray free electron laser (XFEL), ultralow emittance and high current, with reproducibility and precise controllability over energy, are required. It has been shown recently that under certain circumstances the output from laser-plasma-accelerators may be already sufficient to yield the minimum conditions needed for FEL radiation production [29]. However, a substantial increase in electron bunch quality is highly desirable for plasmabased accelerators, in order to increase the brightness of electron drivers for XFEL applications; this increase in brightness enables a dramatic decrease of the FEL footprint by shortening the FEL gain length, thus helping make a truly compact XFEL system [30]. Further, use of lower emittance beams allows shorter wavelengths to be accessed [31]. 
Recently, hybrid plasma accelerators [32] have been described that promise to enable dramatically increased electron bunch quality by employing an underdense photocathode plasma wakefield mechanism [33] - the equivalent of a solid photocathode in radio frequency (rf) photoinjector guns [34]. In particular, this hybrid injector scheme plasma accelerator (also known as Trojan horse injection [35]) may produce bunches with normalized transverse emittance down to the $\epsilon \approx 10^{-2} \mathrm{~mm} \mathrm{mrad}$ level or less. The essential ingredients of this novel concept are an intense, short pulse electron beam driver; a synchronized, relatively low-energy laser pulse; and a gas/plasma combination consisting of at least one low-ionization threshold (LIT) and another higher-ionization-threshold (HIT) atomic component. The electron beam driver is required to drive the plasma wakefield interaction into the blowout regime [36] in the LIT medium. The synchronized laser pulse then follows the driving electron beam and is focused into the blowout cavity where it releases ultracold electrons via localized (within the laser Rayleigh length) ionization of the HIT gas. In contrast to today's laser wakefield acceleration schemes, where the terawatts-power-scale laser pulse sets up the plasma wave, here, the modest intensity laser pulse is required only to release electrons into the electron beam-driven plasma wakefield acceleration, in underdense photoionization action. The separation of function between driving of very large-amplitude plasma waves and the ionization injection between the electron beam and laser beam, respectively, gives a high degree of tenability in this approach.

Figure 1 illustrates this underdense photocathode plasma wakefield acceleration process based on particle-in-cell (PIC) simulations with VORPAL [37]. The electron bunch driver sets up the LIT plasma wave, and the laser pulse sets free electrons around its focus on an axis with ultralow emittance. These electrons are then caught and form a tiny, ultrahigh quality bunch that is copropagating with the plasma wave at the end of the blowout, thus profiting from maximized energy gain.

This scheme has been shown in simulations to yield beams with high current (hundreds of amperes) and unprecedentedly low emittance; these are high brightness beams with very strong promise as new-generation XFEL drivers. For an investigation of the underlying physical mechanisms limiting the performance of this scheme it is instructive to consider first the sources of emittance in conventional photoinjectors, in which electrons are liberated from photocathodes embedded in high field rf cavities. In traditional photoinjectors, there are three contributing factors to the electron beam emittance: those from thermallike effects, time-dependent rf field effects, and collective space-charge forces [38]. When photoinjector systems are optimized, particularly at low charge, one is left with an emittance dominated by thermal effects. This emittance is proportional to the beam rms transverse size $\sigma_{x, y}$ injected

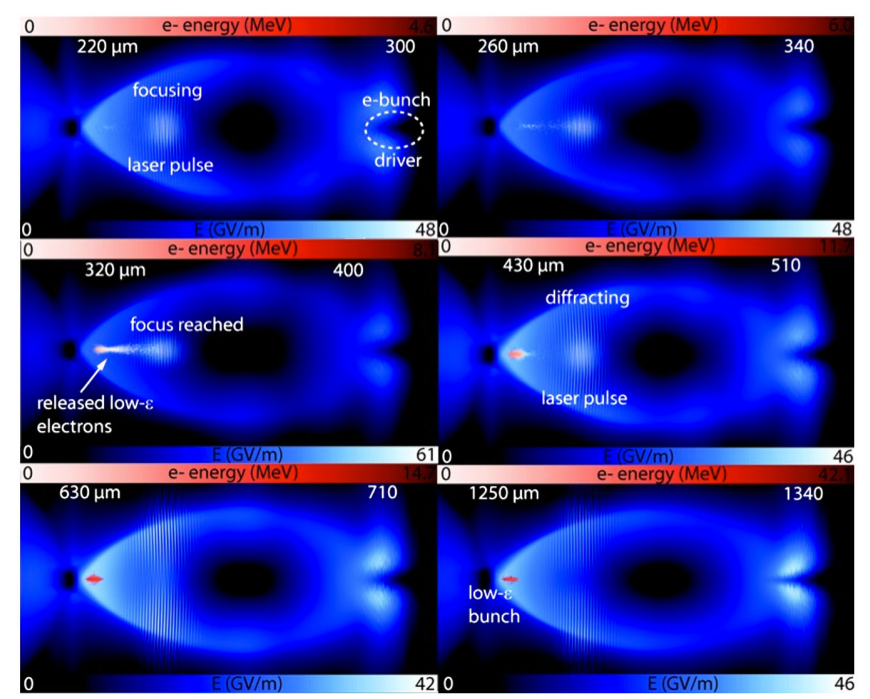

FIG. 1. PIC simulation snapshots of photoionization release, trapping, and acceleration of electrons inside a bunch-driven, self-ionized plasma blowout. The copropagating laser pulse starts to release electrons as it approaches its focus. These low-emittance electrons are trapped at the back of the blowout and gain energy (red color bar). The blue and white colors show the sum of the electric field, which is necessary to indicate the bubble structure as well as the linearly polarized laser pulse electric field.

at the cathode and also the thermal spread in transverse momenta. In a plasma photocathode, an analogous effect to the thermal emittance contribution arises from the transverse momentum obtained during the initial interaction with the ionizing laser pulse. One may profitably view this mechanism as the dressing of the electrons at birth by the canonical electromagnetic momentum associated with the laser. Similarly, there are subsequent timedependent field (both plasma and laser) and space-charge effects to be considered after establishing the minimum laser-induced emittance $\epsilon_{l}$. In Trojan horse plasma wakefield acceleration scenarios, the electrons are accelerated in multi-GV/m fields, thus helping to mitigate space-charge effects through extremely quick transit of nonrelativistic energies. These fields also enable production of few- or even sub-fs-scale duration electron beams with nonnegligible (pC-level) charge and thus yield high current beams needed for application. Also, in direct relevance to the present investigation, the fields support formation of small electron bunch size $\sigma_{x, y}$, permitting minimization of $\boldsymbol{\epsilon}_{x, y}$. All of these characteristics are thus affected by the ionization laser parameters. It is therefore critical to understand the parametric dependences of fundamental processes involved in laser ionization, which occur in the environment of external plasma fields. The multidisciplinary investigation of these issues involves a detailed understanding of a variety of physical effects arising from an impressive number of disparate fields: plasma physics, (relativistic) beam physics, atomic physics, and optical science. 


\section{CONTRIBUTIONS TO THE BEAM EMITTANCE}

We are thus searching to determine the dependences of $\epsilon_{l x, l y}$, which in contrast to the minimum emittance in photocathode devices typically has both strong thermal and time-dependent characteristics. Indeed, laser ionization contributes to factors in the emittance in two proportional ways: through the electron off-axis positions $(x, y)$ and the net momenta $\left(p_{x}, p_{y}\right)$ obtained from the oscillating laser pulse field after release of the electron from the atomic species. Ionization theory is used to provide information about the initial conditions of electrons in phase space, using the analytical fields of the laser near its focus. Subsequent electron trajectories are obtained by solving their equations of motion under the applied forces, using analytical laser fields and simulation-derived plasma plasma fields.

The transverse normalized emittance is defined as $\boldsymbol{\epsilon}_{x}=\left(\left\langle x^{2}\right\rangle_{N}\left\langle p_{x}^{2}\right\rangle_{N}-\left\langle x p_{x}\right\rangle_{N}^{2}\right)^{1 / 2} / m_{e} c$ and $\boldsymbol{\epsilon}_{y}=\left(\left\langle y^{2}\right\rangle_{N}\left\langle p_{y}^{2}\right\rangle_{N}-\right.$ $\left.\left\langle y p_{y}\right\rangle_{N}^{2}\right)^{1 / 2} / m_{e} c$, where $x, p_{x}$ and $y, p_{y}$ are the transverse Cartesian off-axis positions and momenta, respectively, and \langle\rangle$_{N}$ denotes the average over all generated photoelectrons at a given position in the nominal propagation direction $z$. To evaluate the laser-induced momenta, we study the case in which the laser electric field is linearly polarized in the $x$ direction. The momenta of electrons from tunneling are assumed to be negligible [39,40], thus the momentum distribution arises purely from electrons-laser interaction and macroscopic plasma fields.

As an initial approximation, one may posit that most of the ionization processes occur when the oscillating laser electric field peaks, since the ionization rates decrease dramatically as the electric field amplitude decreases. Further, ionization in vicinity of the field maxima corresponds to the vicinity where the initial vector potential $\mathbf{A}_{\text {ini }}=0$. This in turn implies that the canonical momentum in $x$ (and thus the residual momentum left after laser passage) is minimized, as is the associated mean drift energy $\mathcal{E}_{\text {ini }}=e^{2}\left\langle\mathbf{A}_{\text {ini }}^{2}\right\rangle_{T} / 2 m$, where \langle\rangle$_{T}$ denotes taking average over one laser cycle. Here, the laser pulse is linearly polarized in the $x$ direction for the sake of simplicity. Since the electric field of the laser pulse oscillates only in the $x$ plane, there is no initial canonical momentum contribution in the $y$ direction due to the laser pulse, and a nonvanishing $\epsilon_{y}$ arises only from the spatial $(z)$ dependence of the ionization, an effect that is not present in rf photo. This effect, which is accounted for in our calculations below, yields $\epsilon_{y} \ll \epsilon_{x}$. These initial considerations support the possibility of generating ultralow emittance beams.

\section{THEORETICAL MODELING OF PHOTOELECTRON RELEASE}

In the following, the ionization of HIT atoms and characteristics of the photoelectrons released in the blowout region are analyzed in detail theoretically. The spatial distribution of photoelectrons in the focus is discussed on the basis of nonadiabatic ionization theory. Subsequently, the motion of the electrons in the combined laser field and accelerating and focusing wakefield in the blowout regime is analyzed numerically. The motion of photoelectrons is tracked and the development of aspects of the captured beam, in particular, its emittance, are predicted. Because of their superior short pulse capabilities, Ti:Sapphire lasers are ideally suited to ionize HIT atoms for production of the captured beam. In the case of helium as the HIT medium, which from an experimental view is an appropriate choice since it is present as a buffer gas in lithium ovens used for many existing, successful plasma wakefield acceleration experiments [41] and is also an attractive HIT candidate in environments where both LIT and HIT media are gaseous at ambient conditions [35], the minimum required intensity of the ionization laser is of the order of $10^{15} \mathrm{~W} / \mathrm{cm}^{-2}$. A focus size of $w_{0}=4 \mu \mathrm{m}$ is chosen as nominal, which corresponds to a Rayleigh length (and thus roughly the longitudinal extent of the ionization region) of $z_{r}=$ $\pi w_{0}^{2} / \lambda \approx 63 \mu \mathrm{m}$. The laser pulse duration $\tau_{L}$ may also have an effect on the bunch emittance, by spreading the initial launch phases inside of the wave. Taking into account the availability and parameters of typical short pulse Ti:Sapphire lasers, $\tau_{L}=32 \mathrm{fs}$ (FWHM) is considered in all of our design examples, with the exception of a parametric study that explores the effects of laser pulse length explicitly.

The time-dependent electromagnetic laser field amplitude is written as

$$
\begin{aligned}
E_{x}= & E_{0} \frac{w_{0}}{w(z)} \exp \left(-\frac{r^{2}}{w(z)^{2}}\right) \exp \left(-\frac{\left(t-\frac{z-z_{w}}{c}\right)^{2}}{2 \tau^{2}}\right) \cos \phi, \\
E_{z}= & 2 E_{0} \frac{w_{0}}{w(z)} \frac{x}{k w(z)^{2}} \exp \left(-\frac{r^{2}}{w(z)^{2}}\right) \exp \left(-\frac{\left(t-\frac{z-z_{w}}{c}\right)^{2}}{2 \tau^{2}}\right) \\
& \times\left(\sin \phi-\frac{z}{z_{r}} \cos \phi\right), \\
B_{y}= & E_{x} / v_{p h, l}, \\
B_{z}= & E_{z} / v_{p h, l},
\end{aligned}
$$

where $z_{w}$ denotes the focus position, $w(z)=w_{0}\left[1+\left(z-z_{w}\right)^{2} /\right.$ $\left.\left(z_{r}\right)^{2}\right]^{1 / 2}$ is the width of pulse at $z, \phi=k z-\omega t+\frac{r^{2}}{w(z)^{2}} \frac{z}{z_{r}}$ $-\zeta(z)$ is the phase, the laser phase velocity $v_{p h, l}$ is near $c$, and $\zeta(z)=\arctan \left(z / z_{r}\right)$ is the Guoy phase shift. To satisfy Maxwell's equations $\nabla \mathbf{B}=0$ and $\nabla \mathbf{E}=0$, the axial field components are included, although for our chosen laser parameters they are approximately 2 orders of magnitude smaller than the transverse components.

Since electrons tunnel the potential barrier in less than the 50 attosecond [42,43], i.e., on time scales small compared to a laser cycle $\tau_{\mathrm{cyc}} \approx 2.7 \mathrm{fs}$, each ionizing process can be well approximated as instantaneous, and a prompt ionization rate distribution based on the laser intensity as it passes near the focus can be assumed. Further, since the 
electron release should take place relatively close to the center of the blowout to obtain suitable capture dynamics, and both the transverse and the longitudinal electric fields arising from plasma response are near zero, it can be assumed that the plasma wakefield itself plays a negligible role during ionization.

The atomic units system $\hbar=e=m_{e}=1$ is used for our discussion of ionization. For He as the HIT medium, the ionization potential is $I_{p} \approx 24.6 \mathrm{eV}$, and the corresponding ponderomotive energy $U_{p}=I / 4 \omega^{2}$ amounts to $U_{p} \approx$ $60 \mathrm{eV}$ at a laser intensity of $I=10^{15} \mathrm{~W} / \mathrm{cm}^{2}$ and a central wavelength of $\lambda=800 \mathrm{~nm}$. The Keldysh parameter at focus is $\gamma_{k}=\sqrt{I_{p} / 2 U_{p}} \approx 0.45$ for this scenario. The ionization process is dominated by tunneling ionization when $\gamma_{k} \ll 1$, while for $\gamma_{k} \gg 1$, multiphoton ionization plays the leading role $[44,45]$. For tunneling ionization, Ammosov-Deloine-Krainov (ADK) theory [46-50] has been successfully developed to solve for the ionization rate by averaging over laser cycles and is well implemented into particle-in-cell simulation codes [51]. In the present concept, the intermediate Keldysh parameter $\gamma_{k} \sim 1$ indicates that both tunnel ionization and multiphoton ionization are relevant. In such a case, the photoelectron yield from ionization is underestimated by ADK theory, especially with short wavelength laser pulses [52], the use of which may be advantageous for the Trojan horse concept. Therefore, to include multiphoton ionization contribution, additionally nonadiabatic tunnel ionization theory based on Landau-Dykhne adiabatic approximation [53] (termed the Yudin-Ivanov, or YI, model) is taken into account.

The ionization probability rate $\Gamma$ having units of $\mathrm{s}^{-1} \mathrm{~m}^{-3}$ from nonadiabatic theory has, as a function of the laser field, an implicit spatial and temporal dependence [53]. The photoelectron yield is given as

$$
N_{e} \approx n_{\mathrm{HIT}} \sum_{\left(z_{w}-z_{r}\right) / c}^{\left(z_{w}+z_{r}\right) / c} T / 2 \int \Gamma(x, y, \xi, t) \mathrm{d} V,
$$

where $N_{e}$ is the total electron number, $n_{\mathrm{HIT}}$ is the electron density of the HIT medium, and $T$ is one complete laser cycle period. At each moment, the probability rate $\Gamma$ is integrated over the laser pulse volume. In our numerical analysis, the probability is accumulated with a step size of $T / 2$. As we are dealing with ionization near the threshold, the release process is confined to one Rayleigh length around focus.

For example, in order to produce a charge of $Q \approx 1 \mathrm{pC}$ per laser shot, the probability integral indicates that the laser intensity should be $I \approx 1.2 \times 10^{15} \mathrm{~W} / \mathrm{cm}^{-2}$.

Figure 2 shows a snapshot of the spatial ionization probability distribution when the pulse is at focus. The ionization rate is a maximum at the center of the biGaussian laser pulse profile, since the probability decreases sharply with intensity. Note that the ionization rate peaks every half-laser cycle, when the absolute value of the

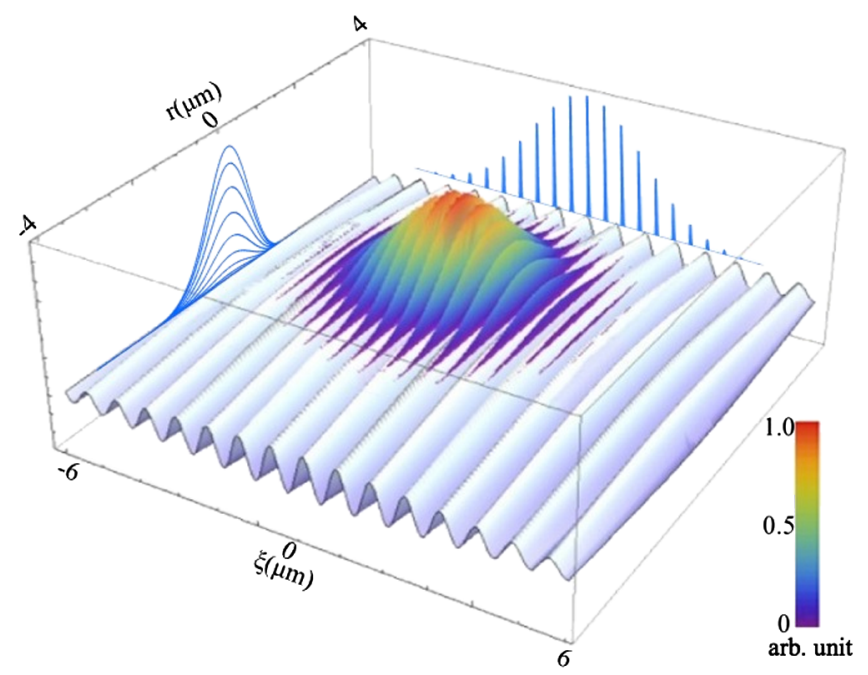

FIG. 2. Visualization of laser-triggered ionization photoelectron yield. The color-coded elevation is the normalized ionization probability rate distribution inside the laser pulse, while the laser pulse profile is shown at the base. The probability distribution is also projected to show longitudinal and axial characteristics.

electric field in the laser pulse and thus the atomic Coulomb potential barrier distortion reach local maxima.

\section{PHOTOELECTRON MOTION IN SUPERIMPOSED LASER AND PLASMA FIELDS}

Next, the electrons released via laser ionization are numerically injected into a laser and plasma field configuration, in which the laser fields are obtained from the prescription given above and the plasma fields derived from 2D particle-in-cell simulations using the PIC code VORPAL. Because of the relevance for the upcoming Facility for Advanced Accelerator Experimental Tests (FACET) experiment E-210: Trojan Horse at SLAC, the driver electron bunch parameters were chosen in the simulation to reflect FACET parameters: the electron energy $E \approx$ $23 \mathrm{GeV}$, the bunch charge $Q \approx 1 \mathrm{nC}$, and the longitudinal and transverse beam sizes are $\sigma_{z}=20 \mu \mathrm{m}$ and $\sigma_{r}=$ $15 \mu \mathrm{m}$, respectively. Thus, the maximum FACET electron beam density is $n_{b}=Q /(2 \pi)^{3 / 2} e \sigma_{r}^{2} \sigma_{z} \approx 8.8 \times 10^{16} \mathrm{~cm}^{-3}$.

While the optimum blowout condition $k_{p} \sigma_{z} \simeq \sqrt{2}$ would indicate a desirable LIT plasma wavelength of $n_{\text {LIT }}=1.4 \times 10^{17} \mathrm{~cm}^{-3}$ (assuming complete LIT medium ionization), such a high density would exceed the electron beam driver density and violate the blowout condition $n_{b} \gg n_{\text {LIT }}$. Additionally, the sum of the laser pulse Rayleigh length and the driver bunch length would exceed the plasma wavelength $\lambda_{\text {LIT }} \approx 89 \mu \mathrm{m}$ in the higher plasma density case. This would imply that either the laser pulse front would interact with the driver bunch, or the electrons released by the back of the laser pulse would not be trapped in the first plasma wave bucket. To mitigate these effects, here a LIT plasma density of $n_{\text {LIT }} \approx$ $5 \times 10^{16} \mathrm{~cm}^{-3}$ is chosen, corresponding to $\lambda_{\mathrm{LIT}} \approx 149 \mu \mathrm{m}$. 
Figure 3 (left) gives color plots of the longitudinal electric field $E_{z}$ and the transverse electric and magnetic field $E_{r}$ and $B_{\phi}$, respectively, as obtained using VORPAL simulations for the bunch-plasma interaction with the parameters discussed above. The driver bunch (not shown) moves to the right in these figures, and the position of the laser pulse (also not included in the VORPAL simulations, which are intended only to furnish self-consistent plasma wakefields driven by the intense electron beam) is indicated with the reddish ellipse. The right-hand side of the figure shows lineouts of relevant fields taken at the three different longitudinal positions in the excited blowout. It can be seen that in the area of interest, the transverse wakefields have excellent linearity and symmetry in the center of the blowout region: the electric radial component $E_{r}$ and magnetic azimuthal component $B_{\phi}$ are proportional to $r$, thus providing for minimum emittance growth due to the wakefield itself. Additionally, the electric longitudinal component $E_{\xi}$ is linear with $\xi$, where $\xi=z-v_{p w} t$, which
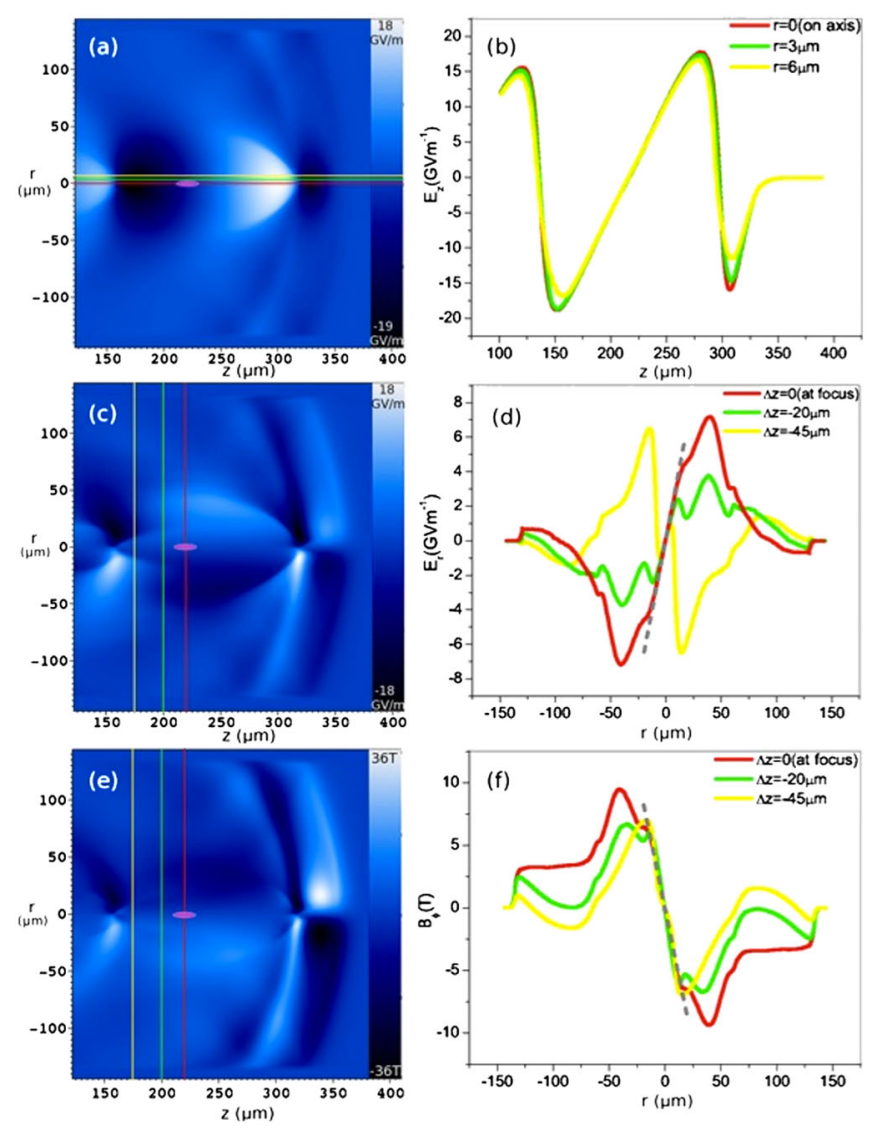

FIG. 3. VORPAL simulation results of the plasma wakefields acting on the released electrons. The left three figures (a), (c), (e) are color plots of the spatial distribution and intensity of wakefields $E_{\xi}, E_{r}, B_{\phi}$, respectively, while the right three figures (b), (d), (f) are lineout plots of the fields at the indicated positions. While the corresponding simulation did not include the laser pulse, the laser pulse position of the laser pulse assumed in the numero-analytical analysis is indicated by the reddish ellipse. is optimum for management of longitudinal oscillations, implying that the longitudinal emittance due to the wakefields is minimized. The motion of the LIT electrons is governed by the Lorentz force relation $d(\gamma m \mathbf{v}) / d t=$ $-e(\mathbf{E}+\mathbf{v} \times \mathbf{B})$ and energy equation $d \gamma / d t=$ $-e \mathbf{v} \times \mathbf{E} / m c^{2}$, where $\gamma$ is the relativistic Lorentz factor $1 / \sqrt{1-v^{2} / c^{2}}$. The time $t$ is normalized to the inverse of the laser frequency $\omega$, and $x, y, z$ are normalized to the inverse of the laser wave number $k$. These choices in turn imply that the velocity $\boldsymbol{\beta}$ is normalized to $c$. Immediately after being released from the HIT atoms, HIT electrons are exposed to both the laser fields $\mathbf{E}_{l}$ and $\mathbf{B}_{l}$ and plasma wakefields $\mathbf{E}_{w}$ and $\mathbf{B}_{w}$, where $w$ and $l$ denote wakefield and laser field. Since the LIT plasma electron density is practically zero inside the blowout, and the ions are quasistatic, there is no plasma response to the laser pulse (which is in diametral contrast to laser wakefield acceleration scenarios). Therefore, the plasma and laser fields can simply be superimposed. Then, when the laser pulse has passed, the electrons are affected only by $\mathbf{E}_{w}$ and $\mathbf{B}_{w}$. For simplicity, $\mathbf{E}$ and $\mathbf{B}$ are normalized to dimensionless (vector potential amplitudes) $\mathbf{a}=e \mathbf{E} / m_{e} \omega c$ and $\mathbf{b}=$ $e \mathbf{B} / m_{e} \omega$, respectively. In summary, the equations of motion equations are rewritten as $\gamma d \boldsymbol{\beta} / d t=\mathbf{V a}+\boldsymbol{\beta} \times \mathbf{b}$ where

$$
V \equiv\left(\begin{array}{ccc}
\beta_{x}^{2}-1 & \beta_{x} \beta_{y} & \beta_{x} \beta_{z} \\
\beta_{x} \beta_{y} & \beta_{y}^{2}-1 & \beta_{y} \beta_{z} \\
\beta_{x} \beta_{z} & \beta_{y} \beta_{z} & \beta_{z}^{2}-1
\end{array}\right)
$$

The laser pulse begins ionization of HIT (He) atoms within roughly a Rayleigh length before it reaches the focal point and the entire ionization process may thus last hundreds of femtoseconds, which leads to betatron phase mixing, and thus emittance growth, through the difference in initiation of the betatron focusing oscillation. Figure 4 shows trajectories of He electrons launched randomly in the path of the laser pulse with probability proportional to the instantaneous, local ionization rate. The top plot shows the variation of velocity in $x$ (the laser polarization) direction, while the bottom plot shows that in $y$ direction. Each electron track initiates with an oscillation due to ponderomotive motion within the laser pulse, which disappears after passage of the laser pulse. Simultaneously, electrons also undergo relatively slow betatron oscillations because of restoring transverse wakefield, which is due almost entirely to ion-column focusing in the electronrarefied region of the blowout. While the oscillatory part of the ponderomotive motion tends to vanish as the laser pulse passes, the laser leaves a remnant transverse "kick." This momentum impulse may be estimated well by calculation of the initial canonical transverse momentum, which is proportional to the laser vector potential at the time and position of the HIT electron release. In contrast, in the $y$ direction, where this vector potential vanishes due to the 

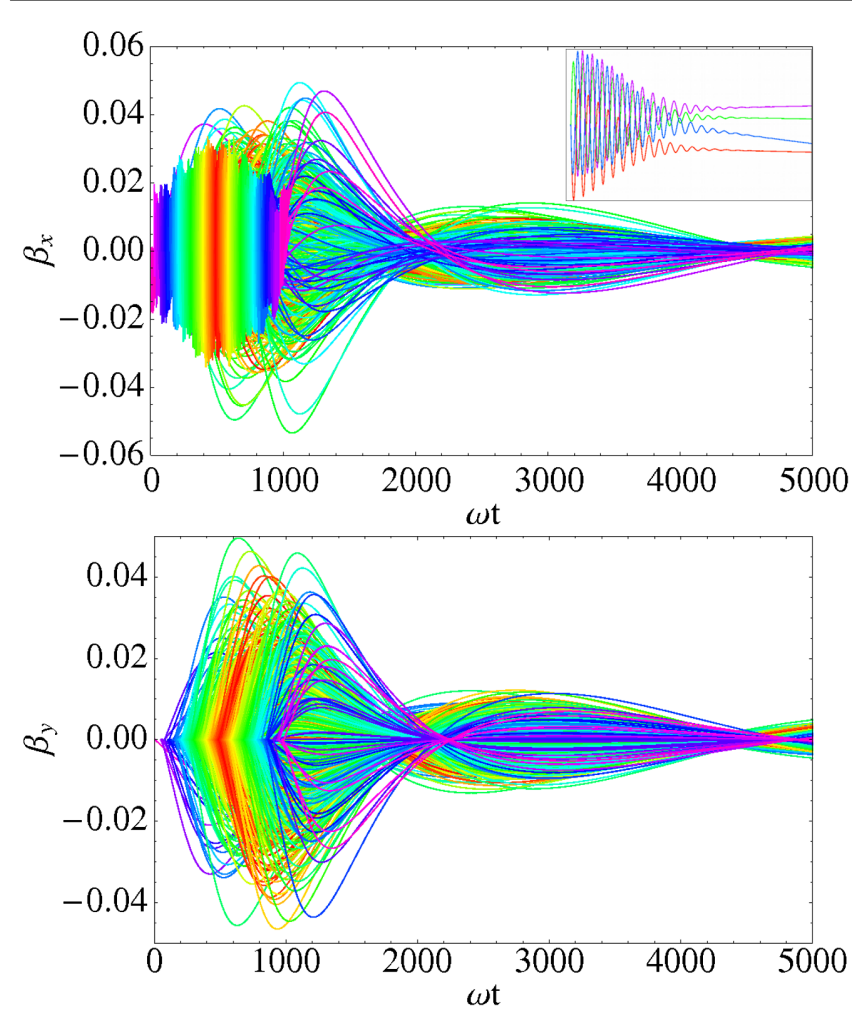

FIG. 4. Electron motion in $x$ (polarization) and $y$ direction are shown in plots at top and bottom, respectively. The inset is a close-up of ponderomotive motion tracks. The tracks are color coded according to electron density from red (maximum) to magenta (minimum).

assumed laser polarization, the beam spread in transverse momentum at a given position in $z$ is only caused by betatron phase mixing. All electron betatron oscillations have a unique starting position. This elimination of laserinduced momentum in the $y$ direction gives the possibility of observing and quantifying phase mixing separately from the direct laser-induced momentum due to the laser seen in the $x$ direction.

\section{EMITTANCE AND BRIGHTNESS RESULTS}

To evaluate normalized transverse beam emittance statistically, we rewrite transverse emittances as

$$
\boldsymbol{\epsilon}_{x} \approx \gamma \beta \sqrt{\left\langle x^{2}\right\rangle\left\langle x^{\prime 2}\right\rangle-\left\langle x x^{\prime}\right\rangle^{2}}, \quad \boldsymbol{\epsilon}_{y} \approx \gamma \beta \sqrt{\left\langle y^{2}\right\rangle\left\langle y^{\prime 2}\right\rangle-\left\langle y y^{\prime}\right\rangle^{2}},
$$

where the ray angles $x^{\prime}$ and $y^{\prime}$ are defined as $v_{x} / v_{z}$ and $v_{y} / v_{z}$, respectively. We investigate the emittance of the beam, which by convention requires use of the position and momentum information of electrons at the same $z$ position, as opposed to a constant time $t$. After the injected beam bunch has completed its interaction with the laser pulse, the beam emittance $\epsilon_{x}$ tends to be stable, as one expects given that the dominant force is a linear ion-focusing transverse wakefield. Thus the only increase in emittance
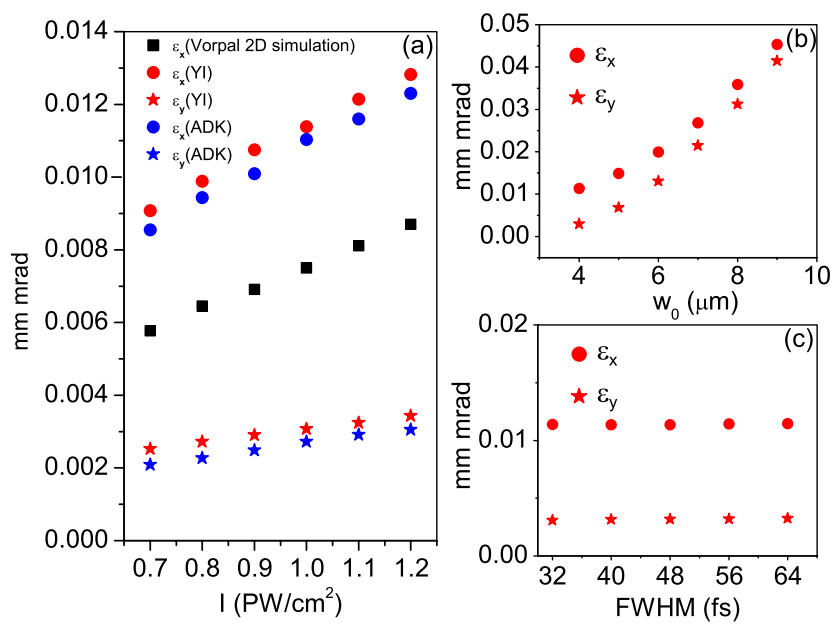

FIG. 5. Laser pulse parametric study of emittance. The plot (a) displays the increase of emittance with laser intensity both in $x$ direction (dot) and in $y$ direction (star). ADK theory (blue) and YI model (red) are compared with results from VORPAL simulation (black square). Note that $\epsilon_{y}$ is excluded due to 2D simulation. At a fixed intensity of $I=1 \mathrm{PW} / \mathrm{cm}^{-2}$, the top and bottom plots on the right show effects of beam waist $w_{0}$ and pulse duration FWHM on emittance, respectively.

would arise from weak chromatic effects due to the finite energy spread in the beam.

As can be seen in Fig. 5(a), the resulting normalized emittance $\epsilon_{x}$ and $\epsilon_{y}$ are of the order $10^{-2}-10^{-3} \mathrm{~mm} \mathrm{mrad}$. The emittance in the dimension orthogonal to the laser polarization $\epsilon_{y}$ is found to be, for the parameters we have chosen, about 1 order of magnitude lower than $\epsilon_{x}$, as it only has a $\epsilon_{\text {mix }}$ component. Both quantities increase approximately linearly with laser intensity $I$ in the parameter range of interest. To explain this result, we emphasize that the beam emittance $\epsilon_{x}$ caused by the laser being polarized in the $x$ direction has two contributions: interaction with laser and betatron phase mixing, which are denoted as $\epsilon_{\text {int }}$ and $\epsilon_{\text {mix }}$, respectively. The contribution $\epsilon_{\text {int }}$ should scale linearly with $I$ because both $x$ and $p_{x}$ due to ponderomotive motion are proportional to the electric field amplitude $E_{0}$. The linearity of $\epsilon_{\text {mix }}$ is numerically obtained by calculating $\left\langle x^{2}\right\rangle=\int x^{2} \Gamma(x, y, \xi, t) \mathrm{d} V \mathrm{~d} t / \int \Gamma(x, y, \xi, t) \mathrm{d} V \mathrm{~d} t$. In the numerical determination of the emittance, both ADK theory and the YI model have been utilized to compare with 2D simulation. We note that ADK theory has been adopted to deal with laser field ionization in the VORPAL framework. Also, space-charge effects have been included in simulation even though it is negligible in our scenario, as discussed below. A possible reason for the lower emittance seen in simulation could be the linearization of the transverse wakefield in modeling. In addition, the emittance predicted by the YI model is slightly higher than that indicated by ADK theory, for the reason that the multiphoton ionization included in the YI model affects the distribution at the extrema of the laser fields more. 
Similarly, we have analyzed the influence of laser waist $w_{0}$ on beam emittance. The emittance from laser interaction can be estimated as $\epsilon_{\text {int }} \propto w_{0}$ while $\epsilon_{\text {mix }}$ is related to $w_{0}^{2}$ due to the $w_{0}^{2}$ factor in the Rayleigh length definition. Therefore, beam emittances grow quadratically with the laser pulse waist [see Fig. 5(b)]. Additionally, as the laser waist increases, the contribution to the emittance due to phase mixing starts to dominate. Consequently, $\epsilon_{x}$ and $\epsilon_{y}$ tend to equalize for large waist size.

On the other hand, the laser pulse duration does not affect beam emittance as significantly as the laser intensity and waist [see Fig. 5(c)]. Certainly, pulse duration plays no direct role in $\epsilon_{\text {int }}$. Also, the degree of phase mixing is determined by Rayleigh length $z_{r}$, given the situation where $z_{r} \gg c \tau_{L}$, not by pulse duration. This implies that for experimental designs the requirement for laser compression to very short pulses is not critical.

When self-field effects due to space charge are included, as will be the subject of future work, the emittance would be expected to exceed the laser-derived emittance alone, particularly at higher bunch charge. It should be pointed out, however, that the existence of very strong ion focusing implies that space-charge effects are secondary, as the distortions in the transverse momentum distribution due to space-charge forces are negligible in amplitude compared to those due to the betatron oscillations arising from the ion restoring force. One may, in the context of near-equilibrium transverse (axisymmetric) beam propagation in the blowout regime, define the ratio of space-charge-to-emittance effects in the transverse rms envelope equation as

$$
R \simeq \frac{I \lambda_{p}}{\sqrt{8} \pi I_{0} \gamma^{3 / 2} \epsilon_{n}}
$$

where $I_{0}=e c / r_{e} \simeq 17 \mathrm{kA}$. This ratio at the exit of a standard photoinjector $(\gamma \simeq 10)$, in which notable space-chargeinduced emittance oscillations must be controlled by the emittance compensation process [54], reaches the range $100-1000$. For our case, in contrast, $R \simeq 0.13$, indicating that the space-charge-induced emittance growth is of secondary concern.

A further implication of the strong focusing and ultralow emittance of the captured beam in the plasma photocathode injector is that the beam density may be extremely large. This space-charge density in turn may cause ion motion, and concomitant emittance growth due to nonlinear ion-derived fields [55]. Indeed, the captured beam density exceeds that of the plasma even at $\gamma=10$ by over 2 orders of magnitude. Nevertheless, since the beam is very short compared to $\lambda_{p}$, and the mass of the LIT ions exceeds that of the electrons by $1.4 \times 10^{3}$, the ion motion should be negligible. In our example, even at high energy $\left(\gamma=10^{4}\right)$, the phase advance of the oscillating ions in the beam's potential well over the beam length $k_{i} L_{b} \simeq 6 \times 10^{-2}$ is negligible.

The evolution and characteristics of the beam's longitudinal phase space have also been studied. Figure 6 shows

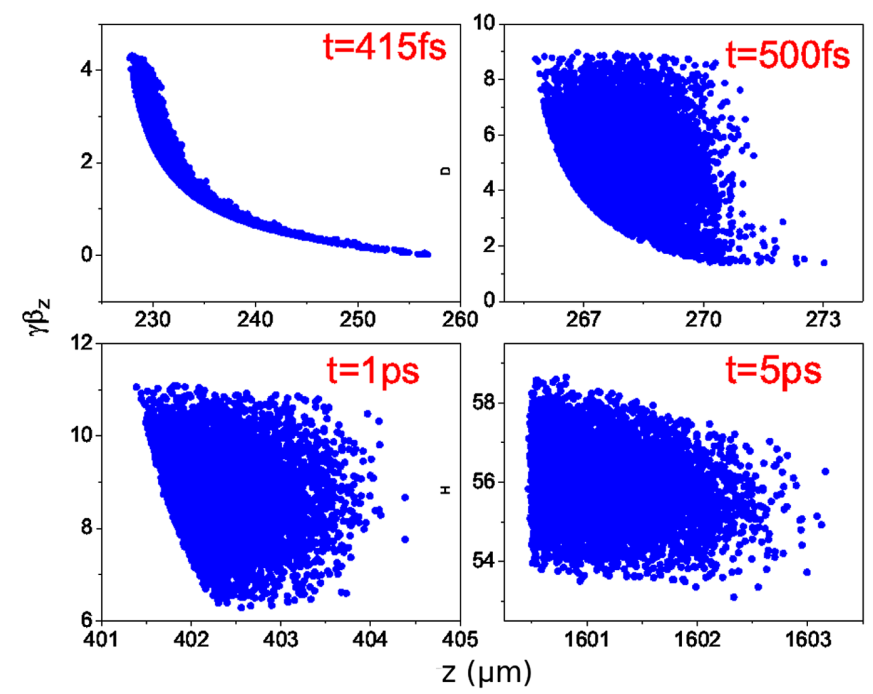

FIG. 6. Snapshots of beam longitudinal phase space at $t=$ $415,500,1,5$ ps. The first figure corresponds to the moment when the ionization is completed.

the evolution of the beam's longitudinal phase space distribution, commencing from the completion of ionization. The observed clockwise rotation of distribution is characteristic of pulse compression as the beam propagates. Beam length $L_{b} \simeq 2.5 \mu \mathrm{m}$ after significant compression, with pC-level charge, which gives a 100 A-level peak current.

The beam brightness, $B \approx 2 I / \epsilon_{x} \epsilon_{y}$, can be estimated accounting for only the laser-induced emittance, as is our emphasis in this paper. Given the longitudinal extent of the beam from modeling and bunch charge yield obtained from Eq. (2), a parametric study of beam brightness is listed in Fig. 7: Frame (a) mostly reflects the effect of charge yield
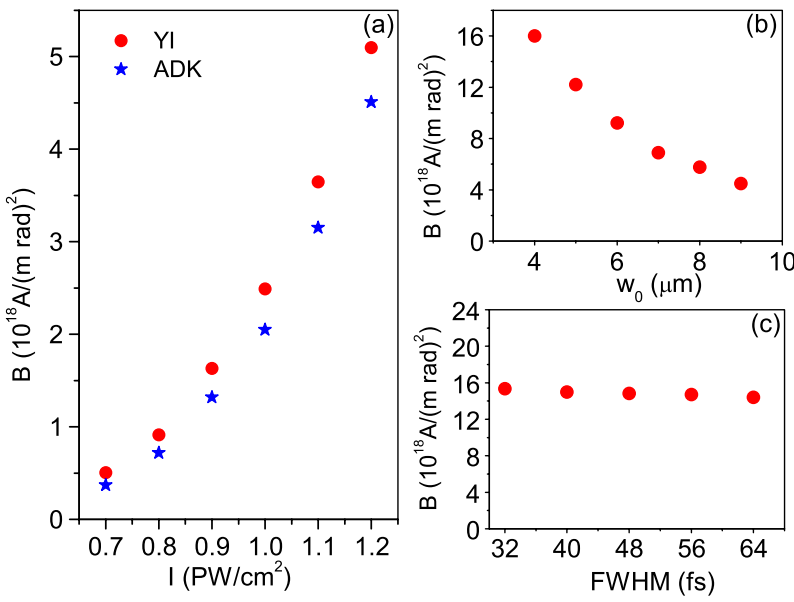

FIG. 7. The dependence of beam brightness on laser intensity, laser waist, and pulse duration are shown in (a), (b), and (c), respectively. 
since emittances are roughly equal from two models. The growth of emittance outweighs the increase of the charge yield in Fig. 7(b). The variation of pulse duration [Fig. 7(c)] does not change either the emittance or the charge yield. The brightness values estimated here exceed that of the state-of-the-art Linac Coherent Light Source photo injector beam [56] by a wide margin, indicating that the beam is a promising candidate for driving a compact FEL, with a gain length notably shorter than that obtained with photoinjectors. In this regard, we note that the rms slice energy spread in our example is approximately $\sigma_{U} \approx 0.5 \mathrm{MeV}$ and so for an XFEL with, for example, $10 \mathrm{GeV}$ energy, the dimensionless gain parameter should exceed the relative slice energy spread, $\rho>\sigma_{U} / U \simeq 5 \times 10^{-4}$. Indeed, given current undulator technology, the beam resulting from our analysis should enable an optimized XFEL system to reach this value of $\rho$.

\section{CONCLUSIONS}

In conclusion, we have investigated the fundamentals of phase space density diluting effects due to the dynamics induced by the ionization laser in an underdense plasma photocathode. This analysis has overcome the need to include information on many different time scales through a hybrid numerical analysis, where the equations of motion of the injected electrons are obtained using the analytical fields of a bi-Gaussion laser pulse, and the plasma wakefields are taken from the results of VORPAL PIC simulations. The atomic physics needed to describe the ionization has been included through use of nonadiabatic intense field ionization theory, permitting us to determine the ionization probability distribution. After ionization, the numerical solution of the LorentzMaxwell equations yields a prediction for the emittance growth caused by laser action; this emittance growth has been evaluated as a function of laser intensity, laser waist, and pulse duration.

The longitudinal evolution of the beam was also analyzed, and the resulting phase space distributions were used to evaluate the beam current and thus the beam brightness. It has been verified that this brightness along with the beam energy spread are sufficient for enabling next generation XFELs. We note that the extremely low emittance in this case permits the use of lower beam energies at a given wavelength and also exploration of yet shorter wavelengths, the sub-Angstrom spectral region. The issues of application of plasma photocathode-derived beams, as well as further space-charge-induced emittance [57], will be addressed in future work.

\section{ACKNOWLEDGMENTS}

We have benefited from valuable discussions with P. H. Bucksbaum and acknowledge simulation resources at the UCLA Hoffman 2 cluster, NERSC and JUROPA. This work is supported by DARPA under Contract No. N66001-11-14197, the U.S. DOE under Contracts No. DE-FG0207ER46272 and No. DE-FG03-92ER40693, by ONR under Contract No. N00014-06-1-0925, by the DFG TR18 and by the European Space Agency under Contract No. 4000102854.

[1] J. B. Rosenzweig, G. Andonian et al., Nucl. Instrum. Methods Phys. Res., Sect. A 653, 98 (2011).

[2] T. Tajima and J.M. Dawson, Phys. Rev. Lett. 43, 267 (1979).

[3] F. Amiranoff et al., Phys. Rev. Lett. 81, 995 (1998).

[4] F. Dorchies et al., Phys. Plasmas 6, 2903 (1999).

[5] P. Chen, J. M. Dawson, R. W. Huff, and T. Katsouleas, Phys. Rev. Lett. 54, 693 (1985).

[6] J. B. Rosenzweig, D. B. Cline, B. Cole, H. Figueroa, W. Gai, R. Konecny, J. Norem, P. Schoessow, and J. Simpson, Phys. Rev. Lett. 61, 98 (1988).

[7] E. Esarey, C. B. Schroeder, and W. P. Leemans, Rev. Mod. Phys. 81, 1229 (2009).

[8] A. Pukhov and J. Meyer ter Vehn, Appl. Phys. B 74, 355 (2002).

[9] S. P. D. Mangles et al., Nature (London) 431, 535 (2004).

[10] C. G. R. Geddes, Cs. Toth, J. van Tilborg, E. Esarey, C. B. Schroeder, D. Bruhwiler, C. Nieter, J. Cary, and W. P. Leemans. Nature (London) 431, 538 (2004).

[11] J. Faure, Y. Glinec, A. Pukhov, S. Kiselev, S. Gordienko, E. Lefebvre, J.P. Rousseau, F. Burgy, and V. Malka, Nature (London) 431, 541 (2004).

[12] S. Y. Kalmykov, A. Beck, S. A. Yi, V. N. Khudik, M. C. Downer, E. Lefebvre, B. A. Shadwick, and D. P. Umstadter, Phys. Plasmas 18, 056704 (2011).

[13] S. Y. Kalmykov, S. A. Yi, A. Beck, A. F. Lifschitz, X. Davoine, E. Lefebvre, V. Khudik, G. Shvets, and M. C. Downer, Plasma Phys. Controlled Fusion 53, 014006 (2011).

[14] S. Banerjee et al., Phys. Plasmas 19, 056703 (2012).

[15] S. Bulanov, N. Naumova, F. Pegoraro, and J. Sakai, Phys. Rev. E 58, R5257 (1998).

[16] H. Suk, N. Barov, J. B. Rosenzweig, and E. Esarey, Phys. Rev. Lett. 86, 1011 (2001).

[17] C. G. R. Geddes, K. Nakamura, G. R. Plateau, Cs. Toth, E. Cormier-Michel, E. Esarey, C. B. Schroeder, J. R. Cary, and W. P. Leemans, Phys. Rev. Lett. 100, 215004 (2008).

[18] K. Schmid, A. Buck, C. M. S. Sears, J. M. Mikhailova, R. Tautz, D. Herrmann, M. Geissler, F. Krausz, and L. Veisz, Phys. Rev. ST Accel. Beams 13, 091301 (2010).

[19] A. J. Gonsalves et al., Nat. Phys. 7, 862 (2011).

[20] D. Umstadter, J. K. Kim, and E. Dodd, Phys. Rev. Lett. 76, 2073 (1996).

[21] E. Esarey, R. F. Hubbard, W. P. Leemans, A. Ting, and P. Sprangle, Phys. Rev. Lett. 79, 2682 (1997).

[22] J. Faure, C. Rechatin, A. Norlin, A. Lifschitz, Y. Glinec, and V. Malka, Nature (London) 444, 737 (2006).

[23] X. Davoine, E. Lefebvre, C. Rechatin, J. Faure, and V. Malka, Phys. Rev. Lett. 102, 065001 (2009).

[24] M. Chen, Z.-M. Sheng, Y.-Y. Ma, and J. Zhang, J. Appl. Phys. 99, 056109 (2006). 
[25] C. McGuffey et al., Phys. Rev. Lett. 104, 025004 (2010).

[26] C.E. Clayton et al., Phys. Rev. Lett. 105, 105003 (2010).

[27] B. B. Pollock et al., Phys. Rev. Lett. 107, 045001 (2011).

[28] J. S. Liu et al., Phys. Rev. Lett. 107, 035001 (2011).

[29] A. R. Maier, A. Meseck, S. Reiche, C. B. Schroeder, T. Seggebrock, and F. Grüner, Phys. Rev. X 2, 031019 (2012).

[30] J. B. Rosenzweig et al., Nucl. Instrum. Methods Phys. Res., Sect. A 593, 39 (2008).

[31] F. H. O'Shea, G. Marcus, J. B. Rosenzweig, M. Scheer, J. Bahrdt, R. Weingartner, A. Gaupp, and F. Grüner, Phys. Rev. ST Accel. Beams 13, 070702 (2010).

[32] B. Hidding, T. Königstein, J. Osterholz, S. Karsch, O. Willi, and G. Pretzler, Phys. Rev. Lett. 104, 195002 (2010).

[33] B. Hidding et al., AIP Conf. Proc. 1507, 570 (2012).

[34] S. C. Hartman et al., Nucl. Instrum. Methods Phys. Res., Sect. A 340, 219 (1994).

[35] B. Hidding, G. Pretzler, J. B. Rosenzweig, T. Konigstein, D. Schiller, and D. L. Bruhwiler, Phys. Rev. Lett. 108, 035001 (2012).

[36] J. B. Rosenzweig, B. Breizman, T. Katsouleas, and J. J. Su, Phys. Rev. A 44, R6189 (1991).

[37] C. Nieter and J. R. Cary, J. Comput. Phys. 196, 448 (2004).

[38] K. Kim, Nucl. Instrum. Methods Phys. Res., Sect. A 275, 201 (1989).

[39] J. L. Krause, K. J. Schafer, and K. C. Kulander, Phys. Rev. Lett. 68, 3535 (1992).

[40] P. B. Corkum. Phys. Rev. Lett. 71, 1994 (1993).

[41] I. Blumenfeld et al., Nature (London) 445, 741 (2007).
[42] PH. Bucksbaum, Science 317, 766 (2007).

[43] A. Landsman, M. Weger, J. Maurer, R. Boge, A. Ludwig, S. Heuser, C. Cirelli, L. Gallmann, and U. Keller, arXiv:1301.2766.

[44] N. B. Delone and V.P. Krainov. Phys. Usp. 41, 469 (1998).

[45] V. S. Popov. Phys. Usp. 47, 855 (2004).

[46] A. I. Nikishov and V.I. Ritus, Sov. Phys. JETP 23, 162 (1966).

[47] A. M. Perelomov, V.S. Popov, and M. V. Terent'ev, Sov. Phys. JETP 23, 924 (1966).

[48] A. M. Perelomov, V.S. Popov, and M. V. Terent'ev, Sov. Phys. JETP 24, 207 (1967).

[49] A. M. Perelomov and V. S. Popov, Sov. Phys. JETP 25, 482 (1967).

[50] M. V. Ammosov, N. B. Delone, and V. P. Krainov, Sov. Phys. JETP 64, 1191 (1986).

[51] D. L. Bruhwiler, D. A. Dimitrov, J. R. Cary, E. Esarey, W. Leemans, and R.E. Giacone, Phys. Plasmas 10, 2022 (2003).

[52] V.-M. Gkortsas, S. Bhardwaj, C.-J. Lai, K.-H. Hong, E. L.F. Filho, and F.X. Kärtner, Phys. Rev. A 84, 013427 (2011).

[53] G. L. Yudin and M. Yu. Ivanov, Phys. Rev. A 64, 013409 (2001).

[54] L.Serafini and J. B. Rosenzweig, Phys. Rev. E 55, 7565 (1997).

[55] J. B. Rosenzweig, A. M. Cook, A. Scott, M. C. Thompson, and R. B. Yoder. Phys. Rev. Lett. 95, 195002 (2005).

[56] R. Akre et al. Phys. Rev. ST Accel. Beams 11, 030703 (2008).

[57] F. J. Grüner, C. B. Schroeder, A. R. Maier, S. Becker, and J. M. Mikhailova. Phys. Rev. ST Accel. Beams 12, 020701 (2009). 